\title{
CONDICIONES DE LA PRADERA SUBMAREAL DE Lessonia trabeculata EN LA ISLA INDEPENDENCIA DURANTE "EL NIÑO 1997-98"
}

\author{
CONDITIONS OF SUBTIDAL FOREST OF Lessonia trabeculata \\ IN INDEPENDENCIA ISLAND DURING "EL NIÑO 1997-98"
}

\author{
Ernesto Fernández ${ }^{1}$, César Córdova ${ }^{2}$ y Juan Tarazona ${ }^{3}$
}

\section{RESUMEN}

En pleno evento "El Niño 1997-98", durante 14 meses, se evaluó in situ, en la pradera submareal de Lessonia trabeculata en la Isla Independencia, los cambios de las características de la especie dominante a una profundidad de $3 \mathrm{~m}$ y los cambios en la distribución vertical de las algas hasta $18 \mathrm{~m}$ de profundidad. Los altos valores de la temperatura del mar, alcanzados en el invierno de 1997, generaron un primer efecto tensionante sobre la especie, llegando muchos individuos a perder sus frondas y desprenderse fácilmente del sustrato. Un segundo efecto tensionante ocurrió en el verano de 1998, cuando los altos valores de la temperatura del mar alcanzados, no fueron soportados por las frondas y se desprendieron, reduciéndose el recubrimiento en un $50 \%$. En marzo los tejidos del tercio superior de los estípites se pudrieron y desprendieron; entre febrero y julio la longitud del estípite se redujo de 150,2 a $31,3 \mathrm{~cm}$ y el diámetro del rizoide de 24,2 a $16,4 \mathrm{~cm}$. La presión de los herbivoros contribuyó a dicha reducción, llegando finalmente hasta una densidad nula en agosto.

La incapacidad de regeneración de nuevos tejidos y órganos, la ausencia de reclutamiento, el modo de desaparición observado entre los 3 y $7 \mathrm{~m}$ de profundidad en octubre de 1998, hace pensar en la posibilidad de la desaparición completa del resto de la pradera de $L$. trabeculata que aún se encuentra a profundidades de 15 a $18 \mathrm{~m}$, ya que carece de frondas y presenta caracteristicas similares a las descritas anteriormente a menor profundidad.

Palabras claves: Lessonia trabeculata, algas pardas, El Niño, submareal, Isla Independencia.

\section{ABSTRACT}

The changes of the dominant species features were evaluated in situ, in Lessonia trabeculata subtidal forest during "EI Niño 1997-98", for 14 months, at $3 \mathrm{~m}$ depth and the changes in vertical distribution of algae until $18 \mathrm{~m}$ depth. The high sea temperature values of winter 1997 generated a first stress effect on the species producing in many of them the loss of fronds and easily detachment from the substrate. A second stress effect occurred in summer of 1998 , when fronds did not support the high sea temperature values reached, reducing covering in $50 \%$. The tissues of the stipe, upper third, were rot and fall down in March. Both, the stipe length reduced from 150,2 to $31,3 \mathrm{~cm}$ and rhizoid diameter from 24,2 to $16,4 \mathrm{~cm}$ among February and July, the pressure of herbivorous contributed to the reduction, and finally the density became worthless in August.

The incapacity to regenerate new tissues and organs, the absent of recruitment, the way of disappearance, observed between 3 and $7 \mathrm{~m}$ depth in October 1998 made real the possibility of complete disappearance of the rest of $L$. trabeculata forest; located from 15 to $18 \mathrm{~m}$ depth, because the lack of fronds, and its presentation of similar characteristics previously described at minor depth.

Key words: Lessonia trabeculata, brown algae, El Niño, subtidal, Independencia Island.

\footnotetext{
1 Univ. Nac. Agraria La Molina, Dpto. Biología

2 Univ. Nac. Mayor de San Marcos, Lab. Ficología

${ }^{3}$ Univ. Nac. Mayor de San Marcos, Lab. Ecología Marina
} 


\section{INTRODUCCIÓN}

Las algas pardas submareales de gran tamaño como Macrocystis pyrifera var. humboldtii, Macrocystis integrifolia y Lessonia sp. se distribuyen e intercalan a lo largo de la costa central y sur del Perú, Acosta (1977), Dawson et al. (1964), Santelices (1980), Acleto (1986), Romero et al. (1988), Tarazona et al. (1988), Ramírez y Santelices (1991), Peters y Breeman (1993), Benavente y Aguirre (1994), Arntz y Fahrbach (1996).

Dichas macroalgas, como productoras primarias, cumplen un importante papel, en la red trófica, según Castilla (1985), Núñez y Vásquez (1987), Vásquez y Buschmann (1997) y proporcionan hábitats para una infinidad de invertebrados y peces Romero $e t$ al. (1988), Mendieta (1997).

En la Bahía Independencia, en la costa central peruana, los fondos duros presentan poblaciones dominantes del bivalvo Aulacomya ater y de las macroalgas Macrocystis pyrifera y Lessonia sp., según Arntz et al. (1982), Soenens (1984; 1985), Romero et al. (1988) y Tarazona et al. (1988). Sólo en los rizoides de Lessonia sp. y debido a la heterogeneidad espacial que estos presentan, proporcionando refugio contra los predadores y lugares de asentamiento larval, se encontró una alta diversidad de macrofauna $\left(\mathrm{H}^{\prime}=3,5\right)$ y un total de 143 especies (poliquetos $39,2 \%$; moluscos $26,6 \%$; crustáceos $25,2 \%$ y otros $9 \%$ ).

Se ha mencionado la ocurrencia, en el Perú, de desapariciones masivas de praderas submareales de macroalgas pardas durante eventos "El Niño" (EN) y su posterior recuperación. Sin embargo, no se ha realizado investigación detallada y a largo plazo sobre el grado de perturbación y recuperación de dichos praderas, en cambio en el hemisferio norte hay varias investigaciones al respecto, incluso a muy largo plazo, como de los autores Tegner y Dayton (1987), Dayton et al. (1992), Schroeter et al. (1995), Tegner et al. (1996; 1997) y Graham (1997).
En el sublitoral las praderas de algas pardas (M. pyrifera) colapsaron en marzo de 1983 y grandes cantidades de éstas fueron arrastradas hacia la orilla; la recuperación de las poblaciones de algas pardas frente a las costas del Perú es muy lenta y se inició a fines de 1985 necesitándose hasta un decenio para que alcancen nuevamente su altitud y extensión originales (Arntz y Fahrbach, 1996).

En agosto de 1986, en la Bahía Independencia los sustratos duros del nivel submareal mostraban todavía muchas rocas desnudas, con poblaciones de baja densidad de individuos jóvenes de las algas $M$. pyrifera y Lessonia sp., en los meses posteriores hubo una rápida proliferación y crecimiento de estas macroalgas, extendiendo su distribución vertical de los 6 hasta los $12 \mathrm{~m}$ de profundidad (Tarazona et al., 1988).

En la presente investigación se realizó el seguimiento de los cambios de las características de la especie dominante y los cambios en la distribución vertical de otras algas en la pradera submareal de L. trabeculata de la Isla Independencia durante 14 meses en pleno evento "El Niño 1997-98". Los resultados documentan la velocidad de aparición, duración e intensidad de la perturbación de la pradera, así como la responsabilidad de los principales agentes tensionantes y plantea las perspectivas.

\section{MATERIAL Y MÉTODOS}

Desde setiembre de 1997 hasta octubre de 1998, mensualmente fueron evaluados, in situ, los cambios de las características de la especie dominante Lessonia trabeculata (densidad, recubrimiento, diámetro del rizoide, longitud del estípite principal, longitud de frondas, condición de los órganos y presión de los herbívoros) a una profundidad de $3 \mathrm{~m}$; también los cambios en la distribución vertical de las algas así como las características del relieve y tipo de fondo hasta $18 \mathrm{~m}$ de profundidad en la pradera submareal que forma la especie. El muestreo fue de tipo no destructivo mediante 
buceo SCUBA y con compresora, siguiendo un transecto perpendicular a la línea de orilla, ubicado en una playa protegida, al lado oriental de la Isla Independencia (14\% $15^{\prime} \mathrm{LS}$ ), ubicada en la Bahía Independencia, Ica, Perú (Fig. 1).

Adicionalmente y en una única ocasión, en setiembre de 1997, después de una temporada de mar muy movido con fuerte oleaje (maretazo), se efectuó la evaluación de la biomasa de las algas varadas en una playa expuesta a mar abierto, en Pan de Azúcar, al lado occidental de la Isla Independencia.

\section{Características del fondo de la playa protegida}

Se realizó un transecto desde la zona intermareal hasta los $18 \mathrm{~m}$ de profundidad $y$ mediante buceo, con ayuda de cuerdas y boyas se determinaron las profundidades a lo largo del transecto, asimismo se registró visualmente la ubicación y el tipo de sustrato (piedras, rocas, arena y conchuelas), así como el grado de movimiento del agua y vaivén de las algas.

\section{Características de la especie dominante Lessonia trabeculata}

Para la toma de la densidad se utilizó una circunferencia de PVC (1/2") de $2 \mathrm{~m}^{2}$, la cual se dejó caer al azar y se realizó tres réplicas, desde la superficie del agua hacia el fondo, se procedió al conteo de individuos de la especie dominante encerrados dentro de la circunferencia.

Simultáneamente durante la determinación de la densidad y con tres réplicas se registró el recubrimiento, estimando el porcentaje del área de la circunferencia de PVC, ocupado por las proyecciones de los estípites y frondas de L. trabeculata.

Al azar y en 10 especímenes de $L$. trabeculata se midió, in situ, el diámetro mayor del rizoide de L. trabeculata, entre los

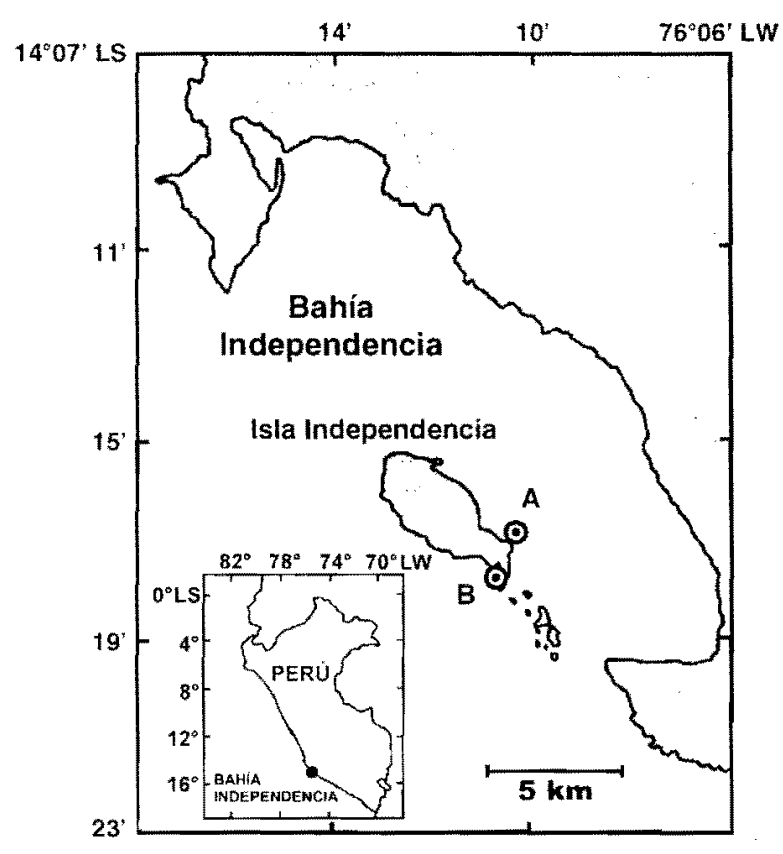

Figura 1. Mapa con la ubicación del área de estudio en las cercanias de la Isla Independencia. A) Playa protegida. B) Playa expuesta. 
hapterios más alejados y diametralmente opuestos.

Siempre al azar y en 10 especímenes de $L$. trabeculata se midió, in situ, la longitud del estípite más largo (principal), desde su base en la unión con el rizoide, por debajo de la primera dicotomía, hasta su extremo distal. En igual forma se procedió con las frondas; se tomó la longitud de 3 frondas del estípite más largo, desde su nacimiento hasta sus extremos.

Se registró, en 10 réplicas al azar, observaciones visuales de la condición de los órganos, considerando las características de color, consistencia, firmeza, pudrición y desprendimiento. Adicionalmente fueron recolectadas algunas muestras de órganos para la observación más detallada en el laboratorio. El registro fotográfico submarino complementó las observaciones.

Se registró la presencia de marcas, raspados, cortes, mordisqueos y excavaciones sobre los rizoides, estípites y frondas, así como los cambios en la morfología. La recolección de muestras así como el registro fotográfico submarino complementaron las observaciones visuales de 10 réplicas al azar.

Adicionalmente, se registró la presencia de fauna sobre los órganos de L. trabeculata y por los alrededores de los individuos.

\section{Distribución vertical de las algas}

Se determinó el límite superior e inferior de distribución vertical de diversas algas entre 0 y $18 \mathrm{~m}$ de profundidad.

\section{Biomasa de algas varadas en playa expuesta}

En setiembre de 1997, luego de un maretazo reciente (mar muy movido y con fuerte oleaje), se realizó una caminata por la orilla de las playas de Pan de Azúcar, ubicadas al lado occidental de la Isla Independencia (cara a mar abierto) y con gran exposición al oleaje, con el fin de evaluar la biomasa de algas varadas. Se identificó la especie dominante, se determinó las dimensiones de la banda de biomasa y el volumen.

\section{RESULTADOS}

En la playa, protegida el relieve del fondo submareal presentó una inclinación muy marcada, en promedio la pendiente fue del $30 \%$. El sustrato de tipo rocoso: el intermareal con piedras redondeadas de 5 a $40 \mathrm{~cm}$ de diámetro, el submareal con piedras y pedrones de hasta un metro de diámetro. Alrededor de los $3 \mathrm{~m}$ de profundidad había algunas salientes de macizo rocoso que llegaron a sobresalir de la superficie del agua. Entre la zona intermareal y los $3 \mathrm{~m}$ de profundidad se observó siempre la fuerte influencia del movimiento del agua sobre el fondo por las mareas, oleaje y maretazos y el frecuente movimiento pendular de las algas, el arrastre y desplazamiento de piedras redondeadas y ocasionalmente el volcado de pedrones de hasta $50 \mathrm{~cm}$. Las piedras y rocas forman un excelente sustrato para los rizoides de $L$. trabeculata.

\section{Características de la especie dominante}

La densidad de L. trabeculata, especie dominante, acusó un decaimiento de diciembre de 1997 hasta agosto de 1998 cuyos valores llegaron a ser nulos $\left(0\right.$ ind. $\left.\times \mathrm{m}^{-2}\right)$.

La densidad de 2.3 ind $\times \mathrm{m}^{2}$ alcanzada en diciembre de 1997 llegó a disminuir en marzo de 1998 hasta 1,5 ind. $\times \mathrm{m}^{-2}$, luego en abril aumentó a 2,17, en mayo disminuyó a 1,16 y en junio volvió a elevarse a 1,5 ind. $\times \mathrm{m}^{-2}$, sin embargo entre diciembre y junio las densidades no fueron significativamente diferentes $(p<0,05)$. En julio la densidad disminuyó bruscamente hasta 0,33 ind. $\times \mathrm{m}^{-2}$, en agosto se hizo nula ( 0 ind. $\left.\times \mathrm{m}^{-2}\right)$ y continuó así en setiembre (Fig. 2).

También pudo observarse en junio y julio $\left(0,33\right.$ y 0,5 ind $\left.\times \mathrm{m}^{-2}\right)$ la presencia de 
individuos caídos sobre el fondo con los discos de fijación despegados del sustrato.

Entre octubre y diciembre de $1997 \mathrm{el}$ recubrimiento de $L$ trabeculata fue del $100 \%$ (Fig. 3), luego disminuyó bruscamente al $50 \%$ en febrero de 1998 y en marzo al $25 \%$. Entre abril y julio el recubrimiento fue disminuyendo lentamente de 20 a $5 \%$. respectivamente. En agosto, se hizo nula por la desaparición de L. trabeculata.

Entre febrero y marzo de 1998 se registró una disminución en el diámetro del rizoide de 24,2 a $18,6 \mathrm{~cm}$, respectivamente (Fig. 4). Luego este valor se mantuvo con una ligera disminución hasta julio $(16,4 \mathrm{~cm})$, sin embargo estas diferencias no fueron significativas $(\mathrm{p}<0,05)$. En agosto los rizoides desaparecieron.

Entre octubre de 1997 y febrero de 1998, a una profundidad de $3 \mathrm{~m}$ se observó un incremento en la longitud del estípite principal de $L$. trabeculata de $109 \mathrm{~cm}$ a $150,2 \mathrm{~cm}$ respectivamente (Fig. 5), luego se registró una ligera disminución hasta abril $(137,9 \mathrm{~cm})$, sin embargo estas diferencias no fueron significativas $(p<0,05)$. A partir de mayo la longitud del estípite fue significativamente menor $(106,2 \mathrm{~cm})$ y en julio se redujo extremadamente a $31,3 \mathrm{~cm}$. En agosto los estípites desaparecieron.

Luego de los desprendimientos de frondas observados durante los calentamientos del agua, las frondas que sobrevivieron y permanecieron unidas a los estípites presentaron una longitud de $21,4 \mathrm{~cm}$ en setiembre de 1997 , de 28 en octubre y de 23,6 en febrero de 1988, estas diferencias no fueron significativas. En marzo la totalidad de las frondas se desprendieron desde su base quedando solamente residuos (Fig. 6).

En agosto de 1996, previo al evento "El Niño", se realizó una inspección general sobre la condición de los órganos, viéndose individuos vigorosos y frondosos de $L$. trabeculata, con una intensa coloración marrón-oscuro, tejidos fuertes y firmes y con una extraordinaria adherencia al sustrato del rizoide. Durante "EI Niño", en setiembre de 1997, se observó sobre la superficie de las frondas, un polvillo crema que las cubría por completo, en octubre las frondas se fueron desprendiendo y en diciembre los extremos

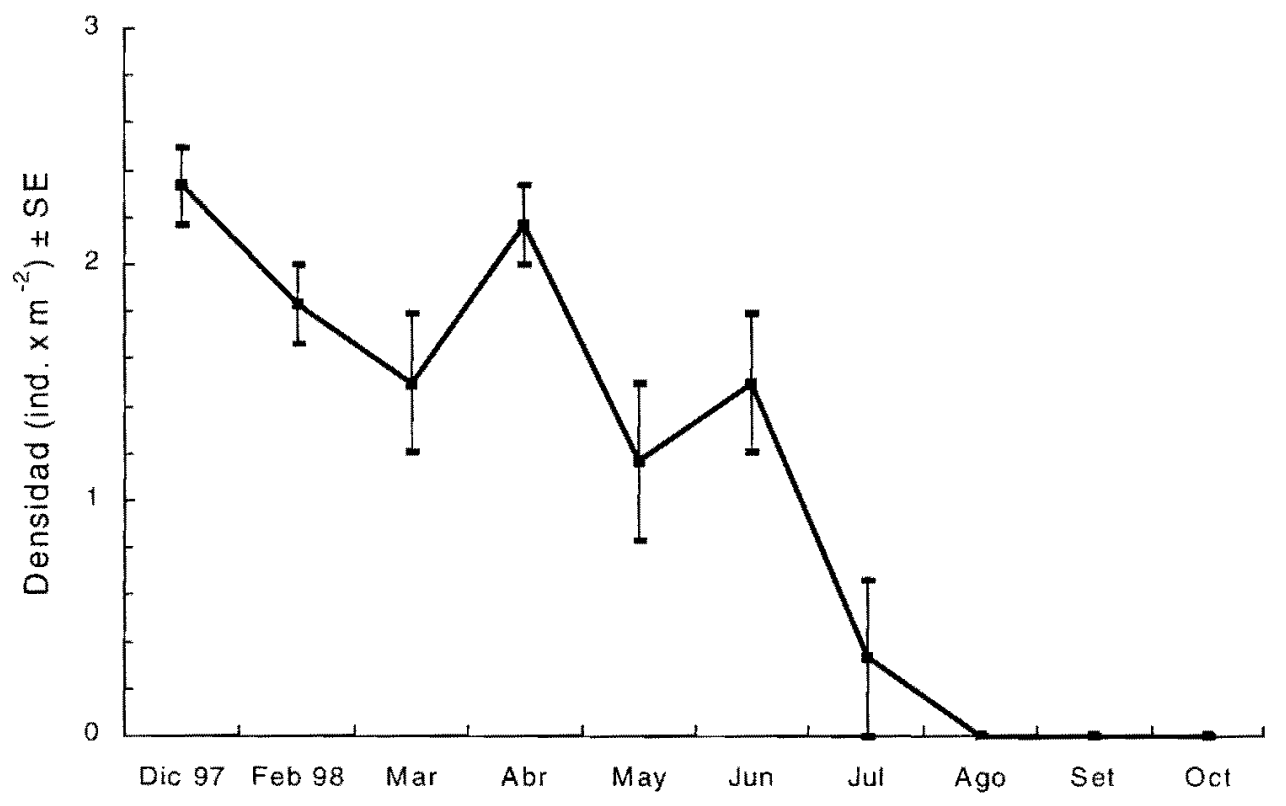

Figura 2. Densidad de L. trabeculata en las cercanías de la Isla Independencia. 


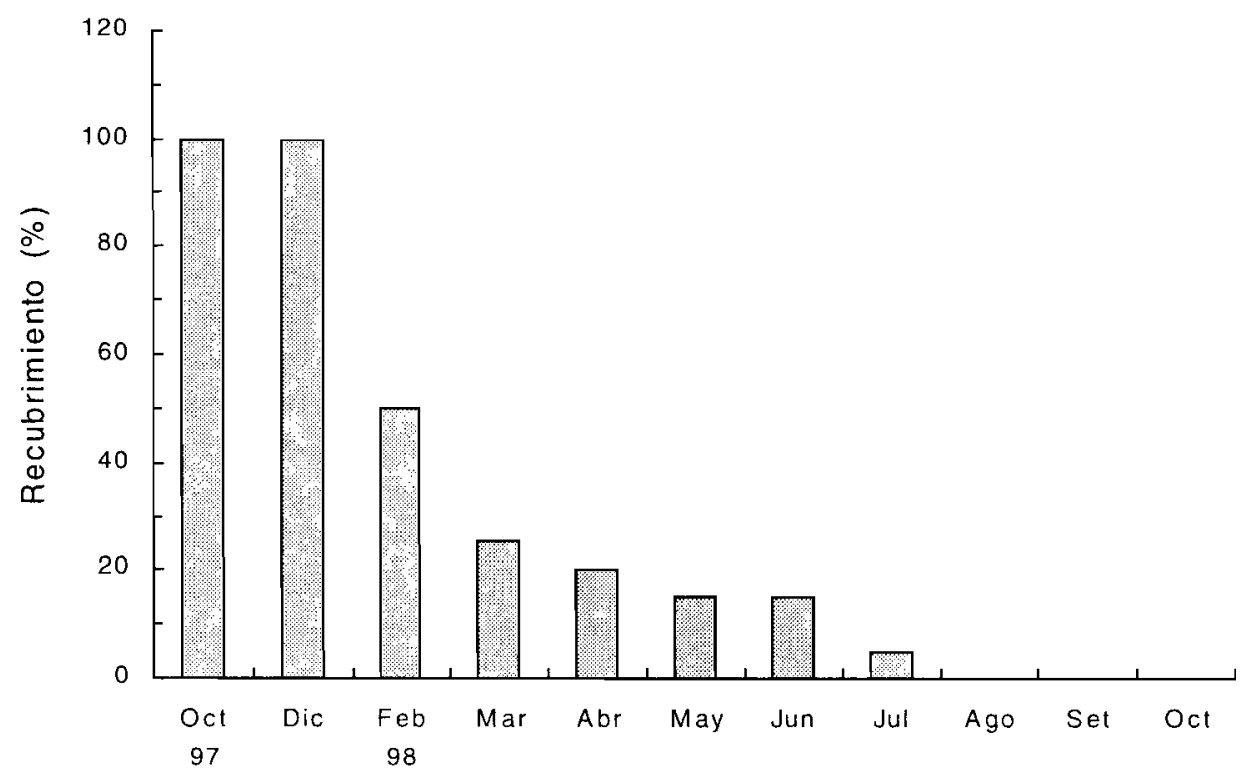

Figura 3. Recubrimiento de $L$. trabeculata en las cercanías de la Isla Independencia.

se tornaron blanquecinos y putrefactos. En febrero de 1998 los estípites presentaron una fuerte decoloración tornándose amarillopálido.

En marzo la mayoría de individuos habían perdido las frondas o sólo presentaban residuos de éstas y los tejidos más externos (meristodermo) del tercio superior de los estípites podridos y desprendidos dejaron expuesta la corteza de un color amarillo intenso. En junio el desprendimiento del meristodermo se extendió hacia los tercios medio e inferior de los estípites y también hacia los discos de fijación, por lo que los individuos $\sin$ frondas quedaron completamente amarillos.

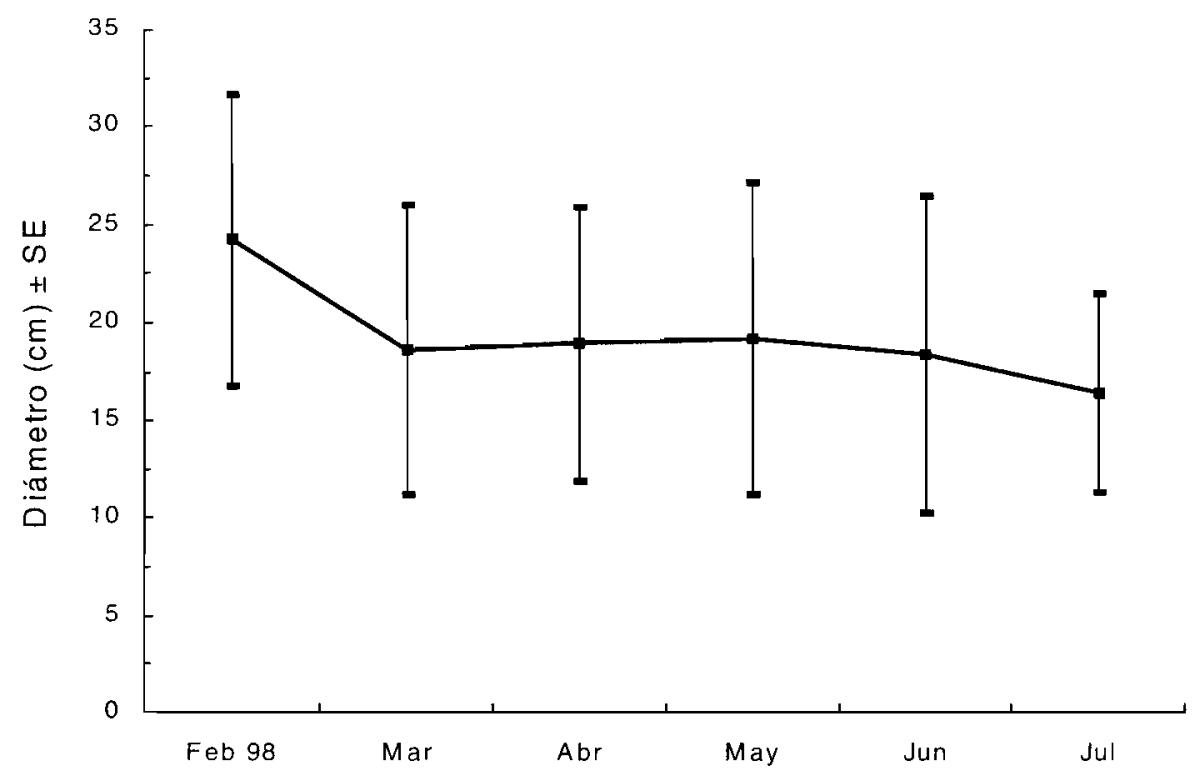

Figura 4. Diámetro del rizoide de L. trabeculata en las cercanías de la Isla Independencia. 


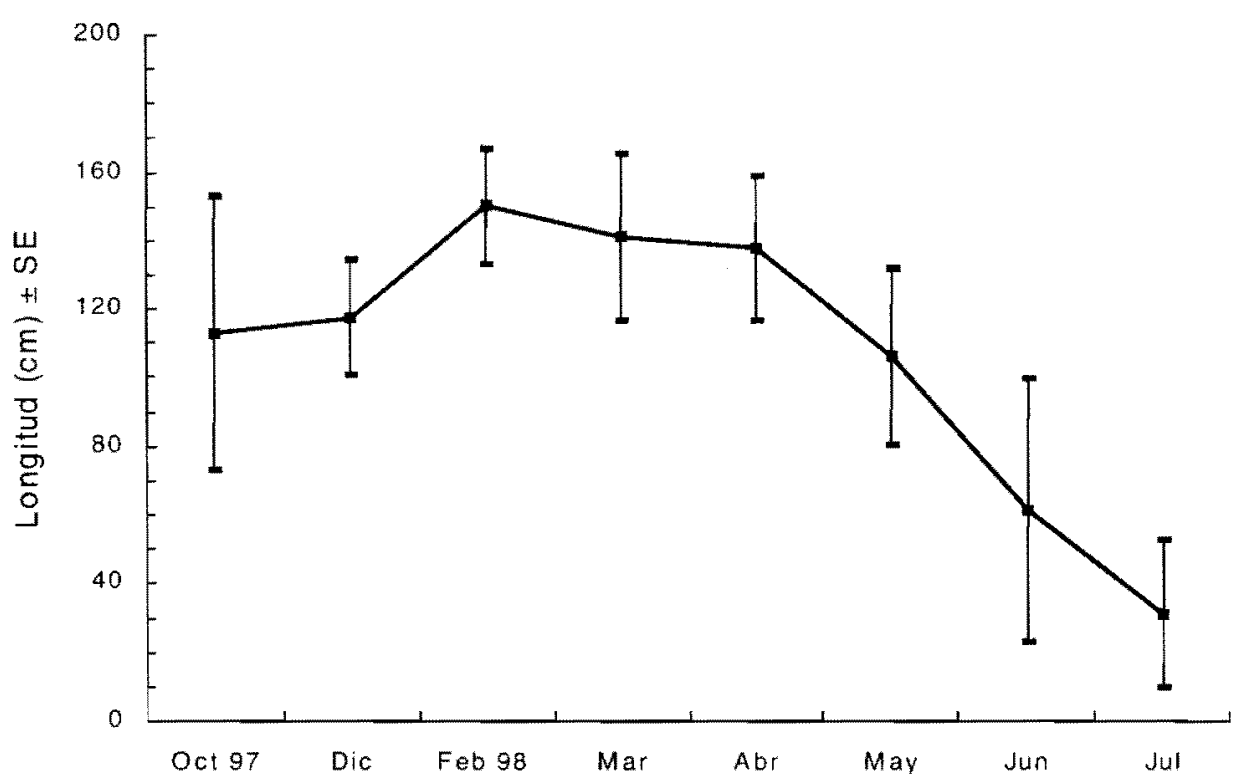

Figura 5. Longitud del estipite de $L$. trabeculata en las cercanias de la Isla Independencia.

La presión de los herbívoros dejó huellas de pastoreo de intensidad intermedia en frondas, estípites y discos de adhesión de $L$. trabeculata en setiembre de 1997. En octubre de 1997 y febrero de 1998, hubo evidencias de un pastoreo más intenso en frondas y estípites (mordisqueos y cortes) así como pequeñas excavaciones en los rizoides. El pastoreo en la base de los estípites generó morfologías alargadas con dicotomías lejanas del disco de fijación y el pastoreo localizado, incluso llegó a cortar desde la base estípites enteros, asimismo la incidencia localizada del pastoreo sobre el rizoide redujo su capacidad de adherencia e hizo que se despegara del sustrato, por lo cual fueron encontrados

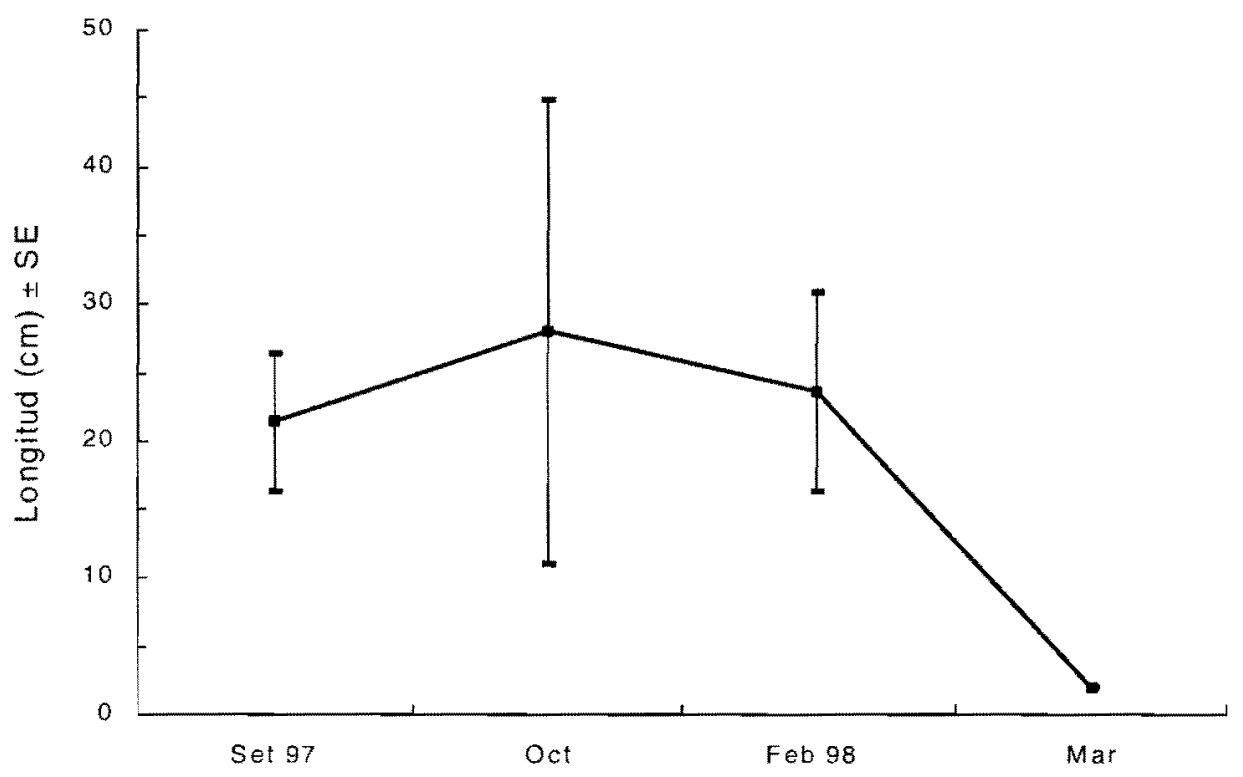

Figura 6. Longitud de frondas de L. trabeculata en las cercanías de la Isla Independencia. 


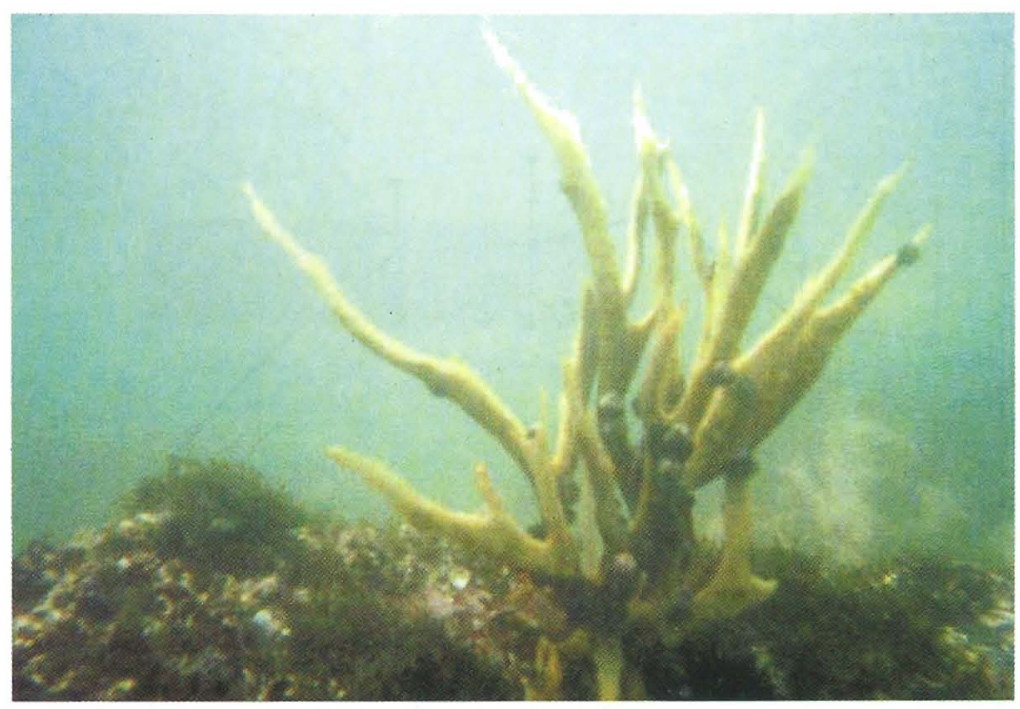

Figura 7. Foto mostrando la fuerte presión de los herbivoros sobre un individuo ya sin frondas de Lessonia trabeculata, en las cercanias de Isla Independencia durante "El Niño 1997-98". El color oscuro de Tegula spp. contrasta con lo claro de los tejidos internos de los estípites que han perdido su cubierta externa.

tumbados sobre el fondo estípites desprendidos con frondas e incluso individuos enteros (con frondas, estípite y rizoide). En marzo continuó el pastoreo sobre estípites y rizoides, en la mayoría de individuos no quedaron frondas, y sólo algunos presentaron una pequeña porción basal remanente de frondas que desaparecieron en abril. En junio, los cortes y mordeduras en los extremos de los estípites dejaron a estos como lanzas afiladas (Fig. 7). También el mordisqueo de los hapterios dejó áreas huecas y rupturas en el rizoide. En julio, una porción del rizoide se despegó del sustrato quedando débilmente adherido y con pequeños trozos remanentes de estípites. En agosto no quedó ya nada.

La fauna observada sobre $L$. trabeculata estuvo compuesta principalmente de gastrópodos (Tegula euryomphalus, Tegula atra y Tegula tridentata) y erizos (Tetrapigus niger y Arbacia spatuligera); en menor proporción los cangrejos ermitaños (Pagurus sp.). Cuando aún existían las frondas se observó la dominancia de Tegula spp. sobre éstas y en menor proporción erizos. Adicionalmente, estos organismos fueron encontrados sobre estípites y rizoides; los pagúridos en la porción inferior de los estípites y también sobre los discos de fijación. Pero cuando L. trabeculata perdió las frondas, la densidad de erizos, caracoles y pagúridos se incrementó notablemente sobre estípites y rizoides. También se observó sobre los individuos de L. trabeculata, tumbados sobre el fondo, gran cantidad de erizos.

Frecuentemente, se observó nadando entre las frondas, estípites y discos de adhesión de L. trabeculata, distintos peces como Cheilodactylus variegatus "pintadilla",

Scartichthys gigas "borracho", Paralabrax humeralis "cabrilla", Oplegnathus insignis "loro", Hemilutjanus macrophthalmos "ojo de uva", Halichoeres dispilus "doncella", Mugiloides chilensis "camote".

La especie dominante, en la distribución vertical de las algas, resultó ser Lessonia trabeculata desde 0,5 hasta $15 \mathrm{~m}$ de profundidad y en algunos casos hasta $18 \mathrm{~m}$ (Tab. 1). Desde el intermareal y luego debajo de L. trabeculata hasta los $3 \mathrm{~m}$ de profundidad hubo densas matas de Glossophora kunthii. Desde abril de 1998 L. trabeculata comenzó a profundizarse a tal ritmo que su límite 
Tabla 1. Rangos de distribución vertical $(m)$ de las algas de la pradera submareal de la Isla Independencia.

\begin{tabular}{|c|c|c|c|c|c|c|c|c|c|c|c|c|c|c|}
\hline Especie & Limites & $\begin{array}{c}96 \\
\text { Ago }\end{array}$ & $\begin{array}{l}97 \\
\text { Set }\end{array}$ & Oct & Dic & $\begin{array}{c}98 \\
\text { Feb }\end{array}$ & Mar & Abr & May & Jun & Jul & Ago & Set & Oct \\
\hline \multirow[t]{2}{*}{ Lessonia trabeculata } & $\mathrm{s}$ & 0,5 & 0,5 & 0,5 & 0,5 & 0,5 & 0,5 & 1 & 1 & 2 & 3 & 7 & 7 & 7 \\
\hline & $\mathrm{i}$ & 15 & 15 & 15 & 15 & 15 & 15 & 15 & 15 & 15 & 15 & 15 & 15 & 15 \\
\hline \multirow[t]{2}{*}{ Glossophora kunthii } & $\mathrm{s}$ & & 0 & 0 & 0 & 1 & 1 & 1 & 0 & 0 & 0 & 0 & 0 & 0 \\
\hline & $\mathrm{i}$ & & 3 & 3 & 3 & 3 & 3 & 3 & 3 & 3 & 3 & 3 & 3 & 7 \\
\hline \multirow[t]{2}{*}{ Costrosas } & $s$ & 0 & 0 & 0 & 0 & 0 & 0 & 0 & 0 & 0 & 0 & 0 & 0 & 0 \\
\hline & i & 1 & 1 & 1 & 1 & 1 & 1 & 1 & 6 & 15 & 15 & 15 & 15 & 15 \\
\hline \multirow{2}{*}{ Antithamnion densum } & s & & & & & & & & & 2 & 3 & 7 & 7 & 7 \\
\hline & $\mathbf{i}$ & & & & & & & & & 15 & 15 & 15 & 17 & 15 \\
\hline \multirow[t]{2}{*}{ Chlorophyta } & s & & & & & & & & & & & & 0 & \\
\hline & $i$ & & & & & & & & & & & & 0,5 & \\
\hline Colpomenia sinuosa & s & & & & & & & & & & & & $\begin{array}{l}0 \\
0,5\end{array}$ & $\begin{array}{l}0 \\
0,5\end{array}$ \\
\hline \multirow[t]{2}{*}{ Laminarial } & s & & & & & & & & & & & & 0 & \\
\hline & $i$ & & & & & & & & & & & & 1 & \\
\hline \multirow[t]{2}{*}{ Florideophycidae } & s & & & & & & & & & & & & 0 & \\
\hline & $i$ & & & & & & & & & & & & 1 & \\
\hline Chondrus canaliculatus & $\mathrm{s}$ & & & & & & & & & & & & & $\begin{array}{l}0 \\
0,5\end{array}$ \\
\hline \multirow[t]{2}{*}{ Chondracanthus chamissoi } & $s$ & & & & & & & & & & & & & 0 \\
\hline & $\mathrm{i}$ & & & & & & & & & & & & & 1 \\
\hline \multirow[t]{2}{*}{ Phyllophora sp. } & $s$ & & & & & & & & & & & & & 16 \\
\hline & $i$ & & & & & & & & & & & & & 17 \\
\hline \multirow{2}{*}{ Cryptonemia sp. } & $s$ & & & & & & & & & & & & & 16 \\
\hline & i & & & & & & & & & & & & & 17 \\
\hline
\end{tabular}

s: limite superior 
superior de un metro, alcanzado en abril, bajó a $3 \mathrm{~m}$ en julio y en agosto a $7 \mathrm{~m}$ de profundidad.

Diversas algas costrosas entre blancas, rosadas, violetas y pardas, presentes desde el intermareal fueron invadiendo los espacios libres, sobre la roca, dejados por los discos de adhesión de L. trabeculata incluso hasta $15 \mathrm{~m}$ de profundidad.

Desde mayo de 1998 se observó sobre los estípites de L. trabeculata una densa capa rojiza que correspondió a la epífita Antithamnion densum, ésta siguió a $L$. trabeculata en su profundización y llegó incluso hasta los $15 \mathrm{~m}$ de profundidad.

Desde setiembre de 1998, algunos pequeños parches de algas verdes, rojas y pardas comenzaron a ocupar espacios someros de cero a un metro de profundidad y las algas rojas ocuparon espacios profundos de 16-17 $m$ profundidad.

En setiembre de 1997, a lo largo de las playas expuestas de Pan de Azúcar, al lado occidental de la Isla Independencia, se encontró una gran cantidad de biomasa de algas varadas y depositadas en la orilla formando una larga banda sobre la playa. En el centro de la banda y en medio de la playa, la biomasa de algas varadas alcanzó una altura de $70 \mathrm{~cm}$ y se estimó un volumen de $957 \mathrm{~m}^{3}$ de $160 \mathrm{~m}$ de playa expuesta.

La mayor proporción de la biomasa de algas varadas se encontró en estado fresco, (recientemente varada) y la especie dominante fue L. trabeculata, aunque también se observó en muy baja proporción algunos rizoides correspondientes a especímenes incompletos de Macrocystis sp. También se encontró individuos de L. trabeculata en estado de descomposición, varados algunas semanas antes; en menor proporción las algas varadas estuvieron desecadas, correspondiendo esto a antiguas varazones.

La mayoría de los especímenes de $L$. trabeculata fueron de gran tamaño (hasta 230 $\mathrm{cm}$ de longitud del estipite principal), y algunos pequeños $(15 \mathrm{~cm}$ de longitud del estípite). Los estípites y el rizoide presentaron marcas de cortes y rupturas. En la mayoría de los especímenes fue notoria la ausencia de frondas o presencia de residuos de éstas.

\section{DISCUSIÓN}

Los altos valores de la temperatura superficial del mar $\left(20,2\right.$ a $\left.19,5^{\circ} \mathrm{C}\right)$ en pleno invierno, entre junio y setiembre de 1997 , generaron un primer impacto sobre $L$. trabeculata ya que muchos individuos llegaron a perder sus frondas se fueron debilitando y fácilmente se iban desprendiendo del sustrato por los movimientos del agua y los fuertes oleajes (maretazos). Esto se manifestó en forma masiva en ambientes expuestos (Pan de Azúcar) y en menor proporción en ambientes protegidos (lado oriental de la Isla Independencia).

No obstante, muchas frondas llegaron a soportar este primer calentamiento del mar e incluso se formaron nuevas frondas y la fotosíntesis continuó en los dos meses siguientes, cuando la temperatura del agua disminuyó un poco (octubre $18,2^{\circ} \mathrm{C}$, noviembre $18,9^{\circ} \mathrm{C}$ ), además, no hubo cambios significativos en la longitud de los estípites.

El segundo impacto se dio en verano (diciembre y febrero de 1998) cuando las altas temperaturas alcanzadas $\left(22,6\right.$ y $22,5^{\circ} \mathrm{C}$ respectivamente) no fueron soportadas por los tejidos de las frondas; se fueron blanqueando, pudriendo y desprendiendo, generando en febrero de 1998 la pérdida del $50 \%$ del recubrimiento de L. trabeculata (aún cuando quedaban frondas con una longitud de 23,6 $\mathrm{cm})$. En marzo, el total desprendimiento de las frondas, dejó a los estípites como único componente del recubrimiento y se redujo al $25 \%$

El segundo calentamiento afectó también los tejidos meristemáticos intercalares ya que el alga fue incapaz de regenerar nuevos tejidos u órganos. La ausencia de frondas y de 
fotosintesis contribuyeron con el debilitamiento generalizado y fueron determinantes en la posterior pudrición y desprendimiento de estípites y rizoides. Mendieta (1997) observó que la poda artificial de las frondas de L. trabeculata en condiciones de alta presión de los herbívoros ocasionaba su muerte.

La disminución significativa de lá densidad de L. trabeculata en forma retardada, recién a partir de junio, se explica por la permanencia de los rizoides que quedaron fijos al sustrato durante varios meses, por la dureza y rigidez de sus tejidos así como a su extraordinaria adherencia. El grosor y la dureza de los estípites también permitieron su larga permanencia, aún cuando fueron incapaces de regeneración alguna.

$\mathrm{El}$ registro de individuos tumbados a $3 \mathrm{~m}$ de profundidad fue incierto, debido a que fueron fácilmente arrastrados a mayores profundidades por el movimiento del agua (con mayor facilidad aún, cuando no habían sido muy pastoreados y eran voluminosos).

Los extremos ramificados (hapterios) de los rizoides fueron más vulnerables al pastoreo, en cambio la porción central fusionada, mucho más gruesa y densa lo fue menos, esto explica la ausencia de diferencias significativas en el tamaño del rizoide hasta julio cuando aún mantenía un $68 \%$ de su tamaño original (aunque con menor volumen por las excavaciones). Un mes después, en agosto, el pastoreo terminó por quebrar los rizoides y debilitar sus puntos de adhesión hacia el sustrato, permitiendo que se despegaran, ante los movimientos del agua. Por otro lado, la reducción significativa en la longitud de estípites, recién a partir de mayo y hasta julio, también evidencia la extraordinaria dureza y capacidad de resistencia de estos tejidos.

Se observó una gran cantidad de erizos y Tegula sp., probablemente fueron los principales responsables del pastoreo 0 consumo de los tejidos en descomposición del alga.

Al respecto Vásquez (1991) observó que la presión de los herbívoros generada por Tetrapigus niger y Tegula tridentata sobre $L$. trabeculata condiciona un determinado fenotipo algal. Vásquez (1990) y Mendieta (1997) también determinaron que cuando los estípites de $L$. trabeculata son podados artificialmente por encima de la primera dicotomía, la fricción entre ellos y con la frondas se reduce, permitiendo una fuerte presión depredadora por parte del erizo negro (Tetrapigus niger) del caracol (Tegula tridentata) y de peces (Aplodactylus punctatus) de tal modo que L. trabeculata es incapaz de remontar las pérdidas ocasionadas por el pastoreo y en 5 ó 6 meses su mortandad alcanza de 50 a $60 \%$, y en 8 a 10 meses $100 \%$.

Por otro lado, Villouta y Santelices (1986) determinaron que en estado adulto $L$. trabeculata normalmente muestra unos pocos estípites ramificados, sólo en sus porciones más altas, debido a que el pastoreo de peces reduce el número y longitud de las frondas.

La dominancia de L. trabeculata sobre otras especies de algas fue notoria, sin embargo los espacios ocupados sobre el sustrato por los rizoides, al quedar libres durante su desprendimiento, fueron ocupados por algas costrosas y dada la dureza de éstas difícilmente podrán ser removidas. La profundización del límite superior de distribución vertical en Glossophora kunthii desde cero a un metro, entre febrero y abril, coincide con las altas temperaturas atcanzadas, pero su poder de recuperación fue rápido, pues en mayo volvió a ocupar espacios desde el intermareal y amplió su línite inferior de distribución vertical hasta los $7 \mathrm{~m}$ de profundidad, dos meses después de que $L$. trabeculata había desaparecido de dicha profundidad. Mendieta (1997) menciona que Plocamium cartilagineum y Glossophora kunthit podrian ser capaces de inhibir el reclutamiento de L. trabeculata limitando el fenómeno sucesional. 
El proceso de desaparición de $L$. trabeculata hasta $\operatorname{los} 7 \mathrm{~m}$ de profundidad que se ha venido presentando hasta octubre de 1998 ha tenido dos etapas: la primera a corto plazo con una gran proporción de desprendimientos de individuos completos y la destrucción de tejidos como respuestas inmediatas a los dos calentamientos y a fuertes movimientos del agua; la segunda etapa, a largo plazo, como consecuencia de la incapacidad de regeneración de nuevos tejidos y órganos, de la ausencia de fotosíntesis, de la pudrición de tejidos y del sobrepastoreo, así como de la ausencia de reclutamientos; nos hace pensar en la completa desaparición del resto de la población de $L$. trabeculata que aûn se encuentra a mayores profundidades (hasta 15 a $18 \mathrm{~m}$ ) ya que carece de frondas $y$ presenta características similares a las descritas a menor profundidad y por lo tanto su desaparición sólo es cuestión de tiempo.

Agradecimientos: Al Consejo Nacional de Ciencia y Tecnología por el financiamiento otorgado a través de la Red sobre Impacto Biológico de los Eventos "El Niño". Al grupo DePSEA de la Universidad Mayor de San Marcos por el apoyo logístico. Al Ministerio de Agricultura por las facilidades brindadas en las instalaciones de la empresa del guano de las islas en la Isla Independencia. A Julio Vásquez por sus recomendaciones en los muestreos y mediciones.

\section{LITERTURA CITADA}

Acleto, C. 1986. Algas marinas del Perú de importancia económica. Publ. Museo Hist. Nat. "Javier Praklo" Ser. Div. (5): 107 pp.

Acosta, P. 1977. Las algas marinas de la provincia de Pisco. Departamento de lca. Perú. Pabl. Maseo Hist. Nat. "Javier Pralo" Ser. B. (82): $\mid-41$

Antz, W.: V. Blaskovich; A. Robles; J. Tarazona: F. Benites y J. Yockteng. 1982. Estudio preliminar de moluscos y crustáceos en la Bahía Independencia (lca, Perú). Inf. Int. PROCOPA - IMARPE: $14 \mathrm{pp}$.

Arntz, W. y E. Fahrbach. 1996. El Niño: Experimento climático de la naturaleza. Fondo de Cultura
Económica, México, 312 pp.

Benavente, M. y G. Aguirre. 1994. Evaluación de la biomasa de poblaciones de Lessonia trabeculata (Phaeophyta. Laminariales) del puerto de Ilo, Perú. Rev. Investig. Cient. Tecnol, Ser. Cienc. Mar. 3: 94-98.

Castilla, 1. 1985. Food webs and tunctional aspects of the kelp. Macrocystix pyrifera, community in the Beagle Channel, Chile. $m$ : W. Siegfred; P. Condy \& R. Laws (eds.). Antartic nutrient cycles and food webs. Springer-Verlab Berlin Heidelberg: $407-414$.

Dayton, P.; M. Tegner, P. Parnell y P. Edwards. 1992. Temporal and spatial patterns of disturbance and recovery in a kelp lorest community. Ecological Monographs 62 (3): 42 l-445.

Dawson, Y.: A. Acleto \& N. Foldvik. 1964. The seaweeds of Peru. Edit. Verlag Von J. Cramer Weinheim. 109 pp.: 82 lám.

Graham, M. 1997. Factors determining the upper limit of giant kelp. Macrocystis pyifera Agardh. along the Monterrey Península, central California. USA. I. Exp. Mar. Biol Ecol. 218 : 127-149.

Mendieta, J.B. 1997. Consideraciones ccológicas para el manejo de poblaciones de Lessonia trabeculata (Villouta \& Santelices 1986) en el norte de Chile. Tesis para optar el grado de Magister en Ciencias del Mar. Univ. Católica del Norte. Fac. de Ciencias del Mar. Chile. $80 \mathrm{pp}$.

Núñez, L. y J. Vásquez. 1987. Observaciones trólicas y de distribución espacial de peces asociados a un bosque submareal de Leswonia trabectalata. Estud. Oceanol. 6: 79-85.

Peters, A. \& A. Breeman. 1993. Temperate tolerance and latitudinal range of brown algac from temperate Pacific South America. Mar. Biol. 115: 143-150.

Ramírez, M. y B. Santelices. 1991. Caúlogo de las algas marinas bentónicas de la costa temperada del Pacífico de Sudamérica. Pont. Univ. Católica de Chile, $437 \mathrm{pp}$.

Romero, L.; C. Paredes y R. Chávez. 1988. Estructura de la macrofauna asociada a los rizoides de Lessonia sp. (Laminariales. Phaeophyta). En: H. Salzwedel y A. Landa (eds.). Recursos y dinámica del ecosistema de afloramiento peruano. Bol. Inst. Mar Peri-Callao Vol. Extraor.: 133-139.

Santelices, B. 1980. Phytogcographic characterization of the tempcrate coast of Pacilic South America. Phycologia 19 (1): 1-12.

Schroeter, S.: T. Dean; K. Thies \& J. Dixon. 1995. Effects of shading by adults on the growth of blade-stage Macrocystis pyrifera (Phaeophyta) during and after the 1982-1984 El Niño. J. Plucol, 31: 697-702. 
Soenens. P. 1984. Crecimicnto y diversidad en comunidades del choro Aulacomya ater Molina, 1782 durante el periodo diciembre 1981 a mayo 1983, en las áreas de Pisco y Huacho. Tesis para optar el Título de Biólogo. Univ. Nac. Agraria La Molina, Lima, Perú, $57 \mathrm{pp}$.

1985. Estudios prelininares sobre el efecto del fenómeno "El Niño" 1982-83 en comunidades de Aulacomva ater. En: W. Arntz; A. Landa y J. Tarazona (eds.). "El Niño", su impacto en la fauna marina. Bol. Inst. Mar Perú-Callao Vol. Extraor.: 51-53.

Tarazona. J.; C. Paredes; L. Romero y S. Guzmán. 1988. La recolonización de las comunidades de mitílidos en la costa central del Perú después de El Niño 1982-1983. En: H. Salzwedel y A. Landa (eds.). Recursos y dinámica del ecosistema de afloramiento peruano. Bol. Inst. Mar Perí-Callao Vol. Extraor.: 115-120.
Tegner, M. \& P. Dayton. 1987. El Niño eflects on Southern California kelp forest communities. Adrances in Ecological Research 17: 243-279.

Tegner, M.; P. Dayton; P. Edwards \& K. Riser. 1997. Large-scale, low-frequency occanographic effects on kelp forest succession: a tale of two cohorts. Mar. Ecol. Prog. 146: 117-134.

Vásquez, J. 1990. Ecological effects ol harvesting Lessonia (Laminariales. Phacophyla) in Central Chile. Hydrobiologia (204/205): $41-47$.

Vásquez, J. \& A. Buschmann. 1997. Herbivore-kelp interactions in Chilcan subtidal communities: A review. Revista Chilena de Historia Natural 70: $41-52$.

Villouta. E. \& B. Santelices. 1986. Lessonia trabeculata sp. nov. (Laminariales, Phacophyta). a new kelp from Chile. Phycologia 25: 81-86. 